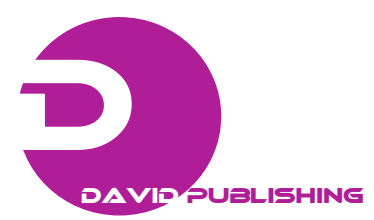

\title{
Fungi Isolated from Insects Collected in Food-Related
}

\section{Facilities in Japan}

Tomoki Sumino*, Masaki Arakawa, Hiromi Mizuhara and Yusuke Matsumi

Teisokasei Co., Ltd., 4005-1 Shiohama, Yokkaichi-shi, 510-0863 Mie, Japan

*Corresponding author's e-mail: tomoki-s@teisokasei.com

\begin{abstract}
Insects are potential mechanical vectors and carriers of pathogenic microorganisms. The fungi (eumycetes), associated with insects, may be harmful to humans as an allergen and cause contamination in the food industry. A total of 47 dead or live insects comprising 21 different families were collected from four study sites (baking factory, rice cracker factory, rice processing factory and central kitchen) in Japan. The fungi isolated from the body surface of household or industrial insect pests were evaluated. In this study, 10 genera of fungi (Cladosporium spp., Penicillium spp., Alternaria spp., Aureobasidium spp., Rhizopus sp., Aspergillus spp., Nigrospora sp., Phoma sp., Curvularia sp., and Fusarium sp.) were isolated from 35 (74.5\%) of 47 collected specimens. These isolated fungi are quite common in human environments. Among the 10 genera of fungi, Cladosporium spp. were the most frequently found in the study sites. There was almost no correlation between insect body sizes and colony forming units (CFU) (Spearman $r^{2}=0.195, P=$ 0.189). Dead and live insects may provide an ideal environment for the growth of fungi in food manufacturing environments. Therefore, good sanitary practices, such as thorough cleaning, should be implemented to eliminate household and industrial insect pests.
\end{abstract}

Key words: Fungi, insects, mechanical vectors, carriers, pathogenic microorganisms and food facilities. 